\title{
La planificación estratégica en las bibliotecas nacionales: su aplicación a la gestión del personal y a la gestión económica
}

\author{
Juan José Fuentes-Romero \\ Universidad de A Coruña
}

\subsection{Resumen}

A partir de unas consideraciones generales sobre la aplicación de la planificación estratégica a las bibliotecas nacionales se estudia la cuestión de la gestión del personal según el estudio realizado por la Biblioteca Nacional del Canadá. Así mismo, se analiza la cuestión de la planificación económica de las bibliotecas nacionales a partir de las pautas señaladas por el Consejo de Europa.

Palabras clave: Bibliotecas nacionales. Planificación estratégica. Gestión.

\subsection{Abstract}

From a general focus on the main problems of the strategic planning applied to the national libraries, this paper presents a study about staff managemennt in the National Library of Canada and, as well, the guidelines of the European Council on economical questions in the libraries, here applied to national libraries.

Keywords: National libraries. Strategic planning. Management.

\section{Introducción}

Si en los últimos tiempos al referirnos al factor humano en cualquier organización ya no se habla de "trabajadores" sino de "recursos humanos" resulta bien evidente que este cambio se ha debido a la constatación del papel principal, esencial diríamos, que el ser humano ha llegado a representar. Desde este enfoque, se considera al otrora llamado "trabajador" a secas como uno de los más importantes recursos de la organización, sea cual sea el tipo y los procesos de trabajo, misiones, objetivos, etc., de ésta.

En modo alguno han sido en esto las bibliotecas una excepción y, en consecuencia, en estos centros objeto de nuestra atención se han ido asumiendo progresivamente unos planteamientos que hoy por hoy ya son comunes en cualquier 
empresa. Entre las razones para este cambio podríamos señalar fundamentalente tres. En primer lugar, desde el punto de vista económico los recursos humanos constituyen, sin lugar a dudas, el más valioso - y, al mismo tiempo, el más caro-de los recursos de cualquier organización. En segundo lugar, las bibliotecas $-\mathrm{y}$ sobre todos las bibliotecas nacionales - consideradas desde el punto de vista de todas sus partes componentes, constituyen operaciones de un altísima inversión económica; lo que exige una notable experiencia en la gestión para garantizar la consecución de sus fines y objetivos dentro de unos costes preestablecidos y asumibles. Por último, la adopción de las más adecuadas estrategias de organización y gestión implica, si se quieren conseguir los objetivos previamente señalados, el mayor respeto y atención a las personas como tales, a sus capacidades profesionales y a sus derechos.

Sea cual sea la teoría de la gestión de la que partamos — desde los estudios de Taylor en adelante-, hoy en día está totalmente admitida la excepcional importancia de dar un adecuado enfoque a la cuestión de los recursos humanos; de modo que temas tales como la motivación, el grado de asimilación de los objetivos de la biblioteca, los indeseables niveles de estrés que un ambiente no deseado pueden llegar a generar, la frustración de quienes ven cómo el sistema de promoción o simplemente de reconocimiento de los méritos propios es injusto, etc., son de vital importancia si se quieren conseguir aquellos resultados por los cuales la sociedad invierte tan fuertes sumas de dinero en las bibliotecas. En cualquier caso, de todos los factores que intervienen en la buena marcha de una biblioteca probablemente sea la adecuada planificación de sus recursos humanos uno de los más importantes, si no el que más, en el mantenimiento de unas condiciones óptimas de trabajo y en el logro de los objetivos que lógicamente se desea alcanzar. Si el factor humano - los recursos humanos, como estamos diciendo - es esencial en la biblioteca, de pura lógica resulta reconocer que el elemento económico marca con su presencia, con su abundancia o, las más de las veces, con su escasez, el tono general de la marcha del centro.

La creciente disminución de la aportación económica por parte de los organismos públicos hace que las bibliotecas tengan que afinar, a veces hasta el perfeccionismo, sus modos de gestión. En más ocasiones de las que se piensa lo esencial de la cuestión para las bibliotecas no está siendo ya el mejor o peor funcionamiento, sino, pura y simplemente, el conseguir unos niveles - por mínimos que sean - de supervivencia.

La planificación, aplicada ahora a la gestión económica de las bibliotecas, resulta absolutamente esencial e insustituible; pues no sólo es problemático que se tengan cada vez menos ingresos, sino que, además, la sociedad exija cada vez más un mayor rendimiento a las aportaciones destinadas a este tipo de centros. 
El estudio que a continuación presentamos muestra dos casos de planificación estratégica aplicada a las bibliotecas nacionales. El primero de ellos se refiere al personal, y está enfocado desde la experiencia de actuaciones de la Biblioteca Nacional del Canadá. El segundo caso trata de la planificación económica contemplada desde la perspectiva de las Pautas del Consejo de Europa sobre legislación y política bibliotecaria.

Parece no obstante conveniente reseñar algo que, por otra parte, consideramos evidente: Con todas las diferencias obvias que queramos, las conclusiones y enseñanzas de este trabajo son perfectamente aplicables a otros tipos de bibliotecas muy diferentes; aquí nos hemos centrado en las bibliotecas nacionales pero las cuestiones de gestión, en este caso de recursos humanos y económicas, son trasladables la mayoría de las veces a otros tipos de bibliotecas en sí enormemente diferentes de las antes citadas.

\section{La planificación bibliotecaria}

De las diversas y numerosas definiciones que se han dado de planificación podemos, a los efectos del presente trabajo, usar la que presentan Kast y Rosenzweig (1987), que afirman:

Planificar es el proceso de decidir anticipadamente lo que se ha de hacer y cómo, e implica la selección de objetivos y el desarrollo de políticas, programas y procedimientos para lograrlo. La planificación resulta ser una actividad integradora que busca aprovechar al máximo la efectividad total de una organización como un sistema de acuerdo con sus metas.

Considerando las estrategias no sólo como uno más de los procesos y vías de la planificación sino, más adecuadamente, como un elemento esencial de actuación a lo largo de todo el desarrollo de la planificación de la actuación de la biblioteca, podríamos entender por dichas estrategias el proceso de decidir sobre los objetivos de la organización, sobre los cambios en estos objetivos, sobre los recursos usados para alcanzarlos y sobre las políticas existentes para dirigir la adquisición, uso y disposición de estos recursos (1).

Cuando en la actual literatura sobre la gestión de bibliotecas se habla de "planificación estratégica" - concepto éste cada vez más usado y difundido, de modo que se está superponiendo y desplazando casi al de "planificación" a secas - normalmente hace mención a la planificación del centro durante un periodo de varios años (Fuentes Romero, 2001, p. 80). Puede también resultarnos operativa y útil la definición de planificación estratégica de Steiner (1969), según el cual:

Planificación estratégica es el proceso de determinar los objetivos principales de una organización así como las políticas y estrategias que van a gobernar la adquisición, uso y disposición para alcanzar dichos objetivos.

Scire. $9: 1$ (en.-jun. 2003) 57-74. 
Para Ferguson (1992) hay tres factores que influyen decisivamente en la planificación estratégica: las características socioeconómicas y los objetivos fundamentales de la organización, sus valores y sus filosofía de gestión y sus fortalezas y debilidades desde la perspectiva de su entorno externo e interno. "Ya que es probable que estos factores varíen debido a los diferentes entornos culturales, socioeconómicos e históricos, es entonces probable que la naturaleza de la planificación estratégica se vea afectada por la localización de la organización".

Al presentar la segunda parte (“Aspectos especiales") de la recopilación de artículos sobre las bibliotecas nacionales correspondiente a 1986-1994, y al referirse a la planificación estratégica aplicada a las bibliotecas nacionales, Line (1995) afirmaba:

La planificación estratégica se ha puesto de moda en las bibliotecas en los últimos años, al igual que ha sucedido en las instituciones del sector público en general. Las razones no están muy lejos: el dinero escasea, los gobiernos tienen otras prioridades en cuanto al gasto, y se busca un mayor control de ese dinero gastado en los organismos del sector público. Estos organismos, que incluyen a las bibliotecas, tienen que justificar cómo gastan el dinero y también han de explicar precisamente cómo se proponen gastarlo en el futuro. Este tipo de control es innecesario en el sector comercial, en el que la necesidad de conseguir beneficios supone una disciplina suficiente. Algunas bibliotecas nacionales se han visto obligadas por sus ministros a preparar planes estratégicos en los que se especifique qué programas intentan poner en marcha en los próximos tres o cinco años; otras han actuado en este sentido voluntariamente.

También, en el mismo trabajo, Line se refiere a la dificultad de llevar a cabo una buena planificación, incluso en condiciones de relativa estabilidad. Citando a Mintzberg (1994), afirma que "hay ahora una reacción general contra la planificación estratégica, partiendo de la base de que no sólo es imposible sino además no deseable". Sin embargo, resulta evidente que las grandes bibliotecas nacionales siguen usando la planificación estratégica como una de las herramientas básicas de la programación de sus actuaciones futuras, máxime en unos tiempos como los actuales en que si hay algo seguro es que casi nada está seguro y, en consecuencia, todo está sujeto a cambios y mutaciones cada vez más extraordinarias.

Sea cuál sea el enfoque desde el que contemplemos la planificación bibliotecaria $-\mathrm{y}$ en modo alguno las bibliotecas nacionales constituyen una excepción a lo que estamos diciendo-, por diverso que sea el tipo de planificación usada siempre se dan en ella tres notas comunes. En primer lugar, la planificación es una actuación global. Se refiere a todo el centro tomado en conjunto, considerado como un todo coherente y homogéneo, de modo que, si es acertado hablar de una planificación de las adquisiciones, del proceso técnico o de la gestión general de las colecciones, no resulta menos acertado el considerar que cada una de estas planificaciones sectoriales no son sino meras sub-planificaciones que actúan no

Scire. $9: 1$ (en.-jun. 2003) 57-74. 
en paralelo sino en convergencia con la planificación global del centro en su conjunto. En segundo lugar, la planificación no es sino una herramienta ante las incertidumbres del futuro. Una buena planificación no es sólo predictiva, intentando averiguar qué va a ocurrir y cuáles van a ser las líneas generales de los cambios que se avecinan. Además, sobre esta actitud más o menos predictiva, la planificación mejor y más útil es la proactiva, es decir, la que, previendo cambios y las líneas generales en que esos cambios van a afectar a nuestra biblioteca, es capaz de servirnos cómo una muy útil herramienta para prepararnos ante esos cambios, para hacerles frente positivamente y con posibilidades de acierto. En tercer lugar, la planificación selecciona las mejores vías de acción. Planificar es elegir, de modo que a partir de una situación nueva y que vemos como posibilidad real en cuanto que va a llegar a afectar a nuestra biblioteca, lo procedente es plantearnos las diferentes propuestas de actuación, de modo que dividida cada propuesta en sus diversos elementos componentes y seamos capaces - mediante la oportuna comparación y confrontación entre dichas propuestas - de decidir cuál es la que en cada momento concreto nos parece la más adecuada en función, siempre, de los objetivos que pretendemos conseguir.

La aplicación a una biblioteca nacional de las ideas y metodología de la planificación estratégica es una cuestión de suyo ardua y compleja que ha sido tratada a fondo por Line en diversos trabajos, entre ellos en su ponencia del congreso mundial de IFLA 1991 en Moscú (Line, 1991). Para Line, debido a una serie de factores diversos, la planificación estratégica presenta en las bibliotecas nacionales una aplicación diferente a la que podría darse en otro tipo de bibliotecas. Entre dichos factores Line señala los cinco siguientes:

- Las bibliotecas nacionales están normalmente mucho más sujetas al gobierno que otras bibliotecas.

- Los recursos de dichos centros pueden verse reducidos de pronto, cambiando drásticamente de año en año de manera impredecible, lo que realmente supone una muy escasa autonomía.

- La clientela de estas bibliotecas está menos definida que la de otras. En consecuencia, la determinación del papel que representan se realiza a menudo más por el gobierno que por sus clientes.

- Las bibliotecas nacionales tienen relaciones y responsabilidades internacionales a un nivel escasamente alcanzado por otros tipos de bibliotecas; consecuentemente, deben funcionar aceptando normas internacionales.

- Al ser una parte del sistema total bibliotecario y de información no pueden actuar aisladamente. Por ello, la elección de las funciones y de las vías a través de las cuáles las realizan puede verse influenciada tanto por lo que otras bibliotecas hacen como por lo que dejan de hacer.

Scire. $9: 1$ (en.-jun. 2003) 57-74. 
Como consecuencia de todo lo hasta aquí expuesto, Line concluye que "las bibliotecas nacionales tienen que planificar bajo la presión del gobierno, a menudo con recursos financieros reducidos, con una clientela incierta, con un amplio abanico de funciones posibles y en el contexto del resto de bibliotecas y de actividades de información de la nación".

Desde que a principios de los 90 - como ya hemos visto - Line llamó la atención sobre la excepcional importancia de la planificación estratégica, éste ha sido un tema recurrente en la atención tanto de los teóricos de estos temas como del sector puramente profesional.

\section{Planificación estratégica y gestión del personal en las bibliotecas nacionales: el caso de la Biblioteca Nacional del Canadá}

Tal vez uno de los problemas más acuciantes que hoy día se presenta a las bibliotecas nacionales es el que se refiere a su personal, pues los recortes y congelación de plantillas constituyen algo que, por desgracia, cada vez es más frecuente en cualquiera de estos centros, incluso en los de los países más desarrollados.

Muchas de estas bibliotecas se ven imposibilitadas para reclutar nuevo personal debido a los pobres salarios, las escasas posibilidades de promoción o las mejores oportunidades que en otros campos se presentan a los graduados en estas materias. Numerosas bibliotecas van viendo cómo se recortan o limitan sus plantillas debido a la política de sus respectivos gobiernos, que, en general y con no muchas excepciones, tienden a una reducción, en cantidad y calidad, de sus funcionarios en todos los departamentos de la actividad pública. Estos recortes y congelaciones de la plantilla están dando por consecuencia que cada vez sea más elevada la media de edad de los técnicos de las bibliotecas nacionales, con las consiguientes dificultades inherentes a la imposible renovación biológica y al hecho de que dicho envejecimiento conlleva lógicamente una falta de iniciativas, de deseos de seguir luchando para estar al día y para adaptarse a las más nuevas tendencias que no dejan de ir apareciendo en un campo de suyo tan contínuamente cambiante cuál es éste de la colección, transferencia y conservación de la información.

A lo dicho hay que añadir la desmoralización de los trabajadores de las bibliotecas nacionales a causa de la presión creciente a la que viven sometidos, a su ya comentada falta de perspectivas de promoción y al hecho de que la escasez misma en el número de profesionales conlleva que los que quedan han de dedicarse cada vez más a labores puramente rutinarias y que no requieren de la enorme experiencia y preparación profesional habitual en la gran mayoría de los especialistas de estos centros de suyo tan complejos.

Parece extenderse cada vez más la idea de que la existencia de un personal fijo, con claras oportunidades de promoción profesional para quienes entran

Scire. $9: 1$ (en.-jun. 2003) 57-74. 
a formar parte de los equipos de trabajo de estos centros, está dando paso a un sistema más fluido de contrataciones temporales que se establece año por año según las disponibilidades crediticias y financieras del centro en cuestión. Numerosas bibliotecas reconocen ya que esto no es un fenómeno temporal y, en consecuencia, están procurando adaptarse adecuadamente a las nuevas circunstancias, utilizando sin ningún tipo de prevención los procedimientos y la metodología de la planificación estratégica y buscando mejores vías en cuanto a la definición, número y tipo de personal que se requiere para hacer frente a la demanda de nuevos servicios con un mínimo de coste. No es ésta, realmente, una tarea fácil de llevar a cabo, pues es difícil concebir de qué manera una biblioteca nacional puede reaccionar adecuadamente ante estos temas cuando se trata de una situación que se viene prolongando ya desde hace bastantes años y respecto a la que no parece haber por parte de los gobiernos ninguna intención de mejorar los niveles de financiación de unos centros a los que constantemente se les están exigiendo una continua puesta al día, así como más y mejores prestaciones, por parte de toda la sociedad.

Nuestro país, España, no constituye desafortunadamente ninguna excepción a cuanto estamos comentando. En este sentido, pueden servirnos las palabras de Smethurst (2001, p. 129) al realizar el análisis global de las bibliotecas nacionales durante el período 1999-2000:

La Biblioteca Nacional de España ha experimentado una considerable pérdida de expertos técnicos dado que el número de su personal ha descendido en años recientes desde los 600 a los 500 miembros. En 1.998 contaba con 475 trabajadores y en 1.999 esta cifra había descendido hasta los 464. Además, parece que el personal técnico puede conseguir mejores salarios y perspectivas de promoción en otros empleos, particularmente en las bibliotecas universitarias, en las de otros ministerios y en las Comunidades Autónomas de España. Esta pérdida de personal está afectando particularmente a los servicios que se ofrecen a los lectores y al proceso técnico de los materiales que van ingresando, ya que por ahora resulta imposible estar al día en su tratamiento. La biblioteca tiene que desarrollar su labor mediante personal conseguido a través de contrato con empresas privadas o a través de estudiantes internos que trabajan supervisados por especialistas.

Otro de los grandes cambios que se están produciendo en el personal de las bibliotecas nacionales está relacionado con las tecnologías de la información y comunicación, el impacto de éstas sobre los métodos de trabajo, la creciente importancia que, cada vez más, se viene concediendo a los servicios a los lectores y, finalmente, la necesidad de desarrollar un personal más flexible y eficaz en la gestión de recursos. Todo esto, en conjunto, ha dado como consecuencia que muchas bibliotecas nacionales estén aplicando las técnicas de la planificación estratégica y concediendo una gran importancia al entrenamiento y a la mayor y mejor preparación en y desde el puesto de trabajo. Entre los requerimientos que

Scire. $9: 1$ (en.-jun. 2003) 57-74. 
aparecen en este nuevo escenario destacan, sin lugar a dudas, los procedentes de tres entornos distintos en su origen pero coincidentes en su influencia sobre las bibliotecas nacionales, cuales son la creciente exigencia del mayor y mejor grado de preparación en el uso de Internet, las cuestiones referentes a los servicios de recepción de usuarios y, finalmente, los temas relativos a la gestión de proyectos.

Algunas bibliotecas, atendiendo a estas cuestiones, han puesto en marcha actuaciones novedosas y originales que están demostrando ser de gran rendimiento y muy eficaces. En este sentido, citamos como ejemplo el caso de la Biblioteca Nacional de Islandia que invita durante cortos períodos de tiempo a colegas de otras bibliotecas nacionales con el propósito de que impartan cursos de entrenamiento o para que estudien la manera en que dicha biblioteca lleva a cabo sus operaciones (Smethurst, 2001, p. 147).

Abundando en esta idea de que uno de los aspectos más importantes en la planificación estratégica de una biblioteca nacional - o más en corto, de una biblioteca sea del tipo que sea- es el que se refiere a su personal, puede resultarnos de enorme interés el informe que en diciembre de 1998 presentó Ms. Gwynneth Evans, Directora General para Programas Nacionales e Internacionales de la Biblioteca Nacional del Canadá ante la Sección de Bibliotecas Nacionales de IFLA (National Library of Canada, 1998). Como punto de partida, Evans señala que durante muchos años las instituciones, tanto del sector público como privado, han reconocido que su recurso más valioso es su gente - el personal que crea los productos y suministra los servicios a los ciudadanos y consumidores - $\mathrm{El}$ nuevo entorno que surge con los cambios debidos esencial, aunque no exclusivamente, a la aparición de las tecnologías de la información y de la comunicación (TICs) afecta enormemente a los requisitos y características que en adelante se va a exigir al personal de las bibliotecas nacionales. En la medida en que el mundo se ha movido inexorablemente hacia una economía basada en el conocimiento y hacia la sociedad de la información, la necesidad de asegurarse de que el personal de cualquier nivel tiene las capacidades y habilidades que permiten a nuestras organizaciones cumplir sus misiones ha llegado a ser mucho más apremiante. En este trabajo de Evans que estamos comentando se muestra la experiencia acumulada durante dos años por la Biblioteca Nacional de Canadá en la definición e implantación entre el personal lo que en la Biblioteca Nacional del Canada se ha llamado el "modelo de las competencias esenciales", método de actuación concebido como una aproximación popular y eficaz para el desarrollo del personal.

Dicho estudio consta de siete partes: los fundamentos de la Biblioteca Nacional para adoptar el método de las competencias esenciales, los objetivos a la hora de definir las competencias esenciales, la metodología utilizada para identificar las competencias de la Biblioteca, los resultados obtenidos y el plan de

Scire. $9: 1$ (en.-jun. 2003) 57-74. 
entrenamiento, el inventario de habilidades y, finalmente, los progresos alcanzados hasta la fecha en la implantación de estas competencias esenciales.

\subsection{Los fundamentos}

Se consideró como punto de partida el trabajo realizado en 1996 para establecer la planificación estratégica de la Biblioteca Nacional del Canadá hasta el año 2001, con la idea de analizar las características de los futuros usuarios de la Biblioteca así como los servicios que se les habría de ofrecer. Surge así el concepto de "habilidades secundarias" (soft skills) como distintas a las tareas y conocimientos reconocidos como "tradicionales" en el trabajo bibliotecario, tales como la catalogación, la referencia y el uso de la tecnología de los ordenadores para proporcionar acceso a la información. Ejemplos de "habilidades secundarias" serían: marketing, negociación, consecución de colaboradores, mantenimiento de una comunicación continua con los clientes y grupos de interés, constitución de equipos, labores de apoyo, gestión de proyectos, preparación de estudios para justificar nuevos servicios, y obtención de fondos. La Biblioteca Nacional llegó a la conclusión de que era tan excepcionalmente importante la definición de las habilidades que se iban a requerir al personal, que estaba justificada la creación de un grupo de trabajo que investigara y presentara unas recomendaciones para implantar un programa de competencias esenciales. Según esto, la Biblioteca creó un Comité de Competencias Esenciales (Core Competencies Committee) que tenía por misión presentar estudios sobre tres temas: a) el perfil de competencias que se consideraban como esenciales para la más adecuada actuación y funcionamiento de la Biblioteca Nacional; b) el plan de entrenamiento para desarrollar en el personal las competencias identificadas: y c) el inventario de habilidades, en el cuál el personal valora las habilidades que se requerirán para su trabajo en cinco años, y compararlas con las que posee actualmente.

\subsection{La metodología}

Este Comité estuvo formado por diversos trabajadores de la Biblioteca que tenían experiencia en la gestión y en las áreas funcionales del Centro y que representaban la diversidad de grupos y niveles ocupacionales, desde los gestores con más experiencia hasta el personal de apoyo. Se usó como material básico la documentación recibida de la sesión de planificación del año 1996 a la que nos referíamos al inicio del epígrafe "Fundamentos", así como los trabajos sobre competencias esenciales llevados a cabo por diversos departamentos del Gobierno canadiense y por otras bibliotecas norteamericanas.

\subsection{La definición}

La primera tarea del comité fue ponerse de acuerdo en una definición del término "competencias". La conclusión fue que las "Competencias son las habili-

Scire. $9: 1$ (en.-jun. 2003) 57-74. 
dades, observables y aplicadas, así como los comportamientos que son esenciales para la actuación personal y para el éxito de la organización”.

Hay dos puntos de esta definición que merecen una especial atención. Por un lado, al definir las competencias que se requieren para el futuro, la Biblioteca estaba buscando un balance entre las obligaciones de los empleados, por una parte, y de sus propios desarrollos profesionales y personales, por la otra. El comité determinó que para que el programa de competencias esenciales tuviera éxito, el personal tenía que sacar en claro que dicho programa les afectaba directamente en su propio crecimiento y desarrollo como individuos y a su puesto de funcionarios públicos. Por el otro, al centrarse en las habilidades y comportamientos, la definición deliberadamente omitía cualquier mención al conocimiento. Dado que muchos trabajos de esta biblioteca nacional, como de cualquier biblioteca nacional o incluso de cualquier biblioteca, conllevan la categoría de conocimientos muy específicos, el comité determinó que la exclusión del conocimiento posibilitaría un concepto más claro de las competencias, especialmente de las esenciales, que iban a ir apareciendo. No obstante, el comité reconoció que todos los trabajos de la Biblioteca Nacional del Canadá tienen un componente de conocimiento y que la habilidad para aplicar el conocimiento requerido es esencial tanto para el trabajo en sí como para el éxito personal. El comité definió las competencias esenciales como "aquellas competencias que tienen una amplia aplicación en la organización y que se relacionan directa o indirectamente con la realización de la misión y objetivos estratégicos de la Biblioteca nacional del Canadá".

Los grupos de trabajo establecidos bien pronto llegaron a la conclusión de que el comité iba a necesitar definir subperfiles separados de competencias para ciertas actividades que, aunque no eran "esenciales" - en el sentido de que no eran requeridas por un grupo numeroso del personal-, sí eran lo suficientemente significativas en términos de proporcionar un apoyo al mandato de la biblioteca en cuanto a garantizar sus propios perfiles. Estas actividades incluían el servicio al público, la gestión y el apoyo de las tecnologías de la información.

Como consecuencia de la actuación de los grupos, el comité elaboró un borrador sobre el perfil de las competencias esenciales, que conllevaba la aplicación a todo el personal de la biblioteca de los tres subperfiles de competencias correspondientes al personal que realizaba actividades especializadas.

\subsection{Los Resultados obtenidos: perfil de competencias}

Los grupos de trabajo establecidos por el Core Competencies Committee identificaron un total de 55 competencias necesarias para el funcionamiento eficaz de la Biblioteca. Para una mayor facilidad de consulta el Comité organizó las competencias en doce secciones. Las ocho primera secciones presentan las competencias esenciales exigibles a todo el personal: competencias en cuanto a

Scire. $9: 1$ (en.-jun. 2003) 57-74. 
las comunicaciones, competencias en cuanto a las tecnologías de la información, competencias respecto a actuaciones ante el cambio, competencias organizativas, competencias para la solución de problemas, competencias para el trabajo en equipo, competencias para la autogestión, y competencias corporativas. Las secciones nueve a doce presentan las competencias requeridas para actividades específicas: servicio, gestión (habilidades con la gente), gestión (habilidades de proceso y organización) y apoyo a los sistemas y tecnologías de la información. A continuación, el documento que estamos analizando presenta una breve descripción de cada una de estas competencias esenciales:

- Comunicación: Una comunicación eficaz es crítica para todo lo que se hace en una biblioteca, por lo que no resulta casual que esta cuestión ocupe uno de los primeros lugares en el listado de competencias. Se entiende que es esencial para individuos y grupos de todos los niveles, tanto dentro como fuera de la Biblioteca Nacional. Las competencias individuales identificadas bajo el epígrafe comunicación incluyen oír, hablar, leer y escribir.

- Tecnologías de la información: Como competencias esenciales, éstas se refieren al uso eficaz del equipo técnico que requiere el trabajo por parte de todo el personal y no sólo de los especialistas. Incluyen la alfabetización informacional - por ejemplo, el uso del teclado y del ratón, de las normas de la biblioteca en este terreno y de programas tales como Windows y de procesadores de texto-, las comunicaciones electrónicas - el correo electrónico y la Intranet de la Biblioteca - y la habilidad para adaptarse a las nuevas tecnologías según estas van siendo introducidas en la biblioteca.

- Las competencias correspondientes a actuaciones ante el cambio pertenecen a la capacidad de cada uno para adaptar su comportamiento personal a las demandas de un entorno de trabajo cambiante. Estas habilidades incluyen la adaptabilidad al cambio, la voluntad para aprender nuevas habilidades y la flexibilidad para trabajar eficazmente en diversas situaciones, con diferentes individuos y grupos y con tolerancia a la ambigüedad.

- Las competencias organizativas incluyen la habilidad para planificar y ejecutar el trabajo con el fin de obtener los resultados deseados: la planificación y gestión del trabajo - por ejemplo, el establecimiento de objetivos-, la gestión del tiempo y el establecimiento de prioridades, el cumplimiento de fechas tope y la gestión del trabajo realizado.

- Se ha definido la solución de problemas como la habilidad para facilitar un eficaz y oportuno resultado en las situaciones de trabajo. Las competencias específicas incluyen habilidades analíticas, habilidades creativas, la aplicación de las habilidades cognitivas propias y la capacidad de comprometerse en la acción - tener iniciativas, asumir riesgos importantes, etc.- .

Scire. $9: 1$ (en.-jun. 2003) 57-74. 
- El trabajo en equipo conlleva la capacidad para trabajar en cooperación con otros para alcanzar metas y objetivos organizacionales comunes. Las competencias específicas incluyen habilidades interpersonales, trabajar eficazmente con otros y fomentar el trabajo en equipo.

- La autogestión se basa en el sentido interno de objetivos y voluntariedad para hacerse responsable de las propias acciones. Las competencias de esta categoría incluyen la automotivación, la iniciativa, la gestión del propio aprendizaje y el comportamiento ético - honestidad y sinceridad y cumplimiento de los compromisos aceptados-.

- Finalmente, las competencias "corporativas" son aquellas que apoyan la misión y mandato de la organización. Para la Biblioteca Nacional del Canadá incluyen la orientación al cliente, el marketing y la promoción - comprensión de los conceptos básicos del marketing y de la promoción en relación con el trabajo individual-, el trabajo dentro del sistema - esto es, la comprensión de la cultura y estructura organizativa, así como la identificación con los objetivos y valores de la organización - y la sensibilidad respecto al entorno - la comprensión del impacto que para la biblioteca nacional representan los cambios políticos, económicos, sociales y tecnológicos procedentes del entorno exterior-.

\subsection{El plan de entrenamiento}

El plan de entrenamiento en Competencias Esenciales de la Biblioteca Nacional se compone de cinco elementos: a) la "parrilla de entrenamiento", que empareja las competencias identificadas con el entrenamiento disponible y con otros recursos; b) una copia del calendario de cursos del equipo de entrenamiento interno; c) una copia del calendario del Servicio de Apoyo a Usuarios de Ordenadores para el entrenamiento técnico interno; d) una lista de consultantes de entrenamiento externos y de los cursos respecto a aquellas competencias que no están gestionadas mediante recursos internos; y e) una lista de recursos de competencias, seleccionada a partir de la World Wide Web, de la colección de entrenamientos de la Biblioteca Nacional y de la colección general de la Biblioteca.

El plan de entrenamiento está diseñado para servir como un recurso continuo para los gestores de la Biblioteca Nacional y para apoyar el desarrollo del personal en cuanto a responsabilidades de trabajo presentes y futuras.

\subsection{El inventario de habilidades}

El inventario de habilidades tiene como objetivo el ser una herramienta de desarrollo que permita a cada trabajador individual gestionar su trabajo y a sí mismo en los términos de las competencias requeridas. Una vez adquirido, este conocimiento va a ayudar al personal a identificar tanto las áreas en las que nece- 
sita mejorar sus habilidades en ese momento como en el futuro. Para cada competencia del perfil el personal valorará en una escala de uno a cinco el nivel hasta el cuál ellos piensan que poseen dicha competencia en ese momento. También valorarán, en columnas separadas, el grado hasta el cuál se requiere cada competencia en el trabajo, tanto entonces como durante los cinco próximos años.

La Biblioteca Nacional no está usando el inventario de habilidades como una parte del proceso de revisión de las actuaciones del personal. Más bien, lo contempla como un instrumento de desarrollo del personal, tanto para cada individuo como para la organización en su conjunto. Todas las respuestas a este inventario son tratadas confidencialmente, y se tabulan y analizan solamente a nivel corporativo, para desarrollar planes de entrenamiento a largo plazo; no individualmente.

\subsection{Los avances}

Hasta el momento en que se hace público este trabajo de Evans se ha distribuido entre el personal, para su información y referencia, el perfil de competencias esenciales, así como los subperfiles y el plan de entrenamiento.

Las respuestas al Inventario van a servir para llenar los vacíos existentes entre las habilidades que el personal posee ahora y las que se requieren en su trabajo desde aquí a cinco años.

La conclusión principal de este estudio incide en la importancia esencial que para cualquier otra biblioteca, sea del tipo que sea, y para cualquier institución tiene su personal, sus recursos humanos. En este sentido se afirma que el cambio desde los 90 hacia el siglo XXI en el que ya nos hallamos ha hecho que las bibliotecas sean más conscientes de que tanto el personal profesional como el de apoyo es fundamental en cuanto a la gestión del conocimiento y desarrollo de las habilidades necesarias para sostener los servicios y recursos de información.

Como vemos es éste un estudio plenamente recomendable por su carácter sintético y globalizador respecto a las diversas cuestiones que atañen al personal de una biblioteca nacional. En no menor medida entendemos que resulta justamente destacable la idea básica que presenta según la cuál los recursos humanos de cualquier biblioteca o lo que es lo mismo, su personal, no tienen más remedio, velis nolis, que adaptarse al nuevo escenario que se presenta en este principio del siglo XXI y del tercer milenio.

No sólo la imparable irrupción de las TIC sino la globalización y los cambios sociales, económicos y políticos que cada día aparecen requieren de un nuevo personal consciente de que su capacidad de adaptación — tanto a nivel individual como en cuanto que forman un colectivo organizado, que junto con la colección de materiales constituye indudablemente el más importante de los recursos de la biblioteca - es la mejor de las garantías para que estos organismos pluricente-

Scire. $9: 1$ (en.-jun. 2003) 57-74. 
narios que son las bibliotecas nacionales sean capaces de sobrevivir y de seguir prestando a la sociedad, que con su esfuerzo económico y con su apoyo las mantiene, los servicios de todo tipo que se le requieren.

\section{La planificación económica de las Bibliotecas Nacionales: Las Páutas del Consejo de Europa}

Sin lugar a dudas uno de los problemas principales y de mayor envergadura, si no el mayor de todos, a los que se enfrenta hoy día una biblioteca nacional es el económico. El proveedor básico de recursos de estos centros suele ser, casi sin excepciones, el gobierno del correspondiente país, lo que conlleva el estar siempre a expensas de las alteraciones presupuestarias de cada ejercicio económico. Lo normal en los último años, sobre todo a partir de la gran crisis económica de fines de los setenta, ha sido una disminución continua y constante de las cantidades que se asignan a los servicios culturales en general y a las bibliotecas nacionales más en particular. También es de justicia recoger aquí la perspectiva un tanto optimista que plantea Smethurst (2001, p. 146) en su último informe sobre la situación de las bibliotecas nacionales europeas. Según dicho autor "En términos de financiación real parece haber una cierta mejora en algunas de las bibliotecas recogidas en el informe".

A lo anteriormente señalado hay que añadir el aumento en las diversas partidas presupuestarias de las bibliotecas, tanto en lo que se refiere al mantenimiento de las operaciones corrientes en cada centro como en cuanto a los materiales que hay que adquirir, los servicios y, muy especialmente, el personal, que resulta ser la más cara de dichas partidas presupuestarias.

Conviene no olvidar que en el caso de las bibliotecas nacionales nos encontramos en la mayoría de los casos, al menos en los países más desarrollados, con unos macrocentros que suponen gastos a veces desmesurados. Entre las causas de estos gastos enormes por parte de las más importantes bibliotecas nacionales podríamos citar las enormes dimensiones de sus edificios e instalaciones, sus inmensas y valiosísimas colecciones de materiales, la enorme y compleja gama de servicios que estas bibliotecas proporcionan a sus usuarios - lo que posiblemente supone un gasto muy por encima sobre cualquier otra partida presupuestaria - , y la ingente cantidad de miembros que forman su plantilla de trabajadores, a lo que hay que añadir, en lo cualitativo, que muchos de esos trabajadores son - o deberían ser- especialistas altamente cualificados.

Al continuo crecimiento de las colecciones y servicios año tras año hay que añadir que una biblioteca nacional que realmente quiera estar en punta de lanza en cuanto a la atención a sus usuarios no tiene más remedio que gastar sumas, a veces considerables, en la realización y desarrollo de estudios respecto a la 
bondad, eficacia y eficiencia de los servicios que suministra a dichos usuarios, ya sean estos reales o potenciales.

Desde esta consideración general sobre los gastos a los que cualquier biblioteca nacional ha de hacer frente habría que señalar en primer lugar los que se refieren al edificio e instalaciones. Según esto, pueden resultar paradigmáticos, entre otros, los casos de las bibliotecas nacionales de Francia y del Reino Unido de Gran Bretaña, en los que la construcción de las nuevas dependencias han supuestos partidas astronómicas de millones y millones de euros (2).

La irrupción masiva de las ya no tan nuevas tecnologías de la información y de la comunicación (TIC) ha supuesto otra de las continuamente crecientes partidas de gastos, no sólo debido al muchas veces altísimo precio de los programas de gestión y control de la información (software) sino al no menos alto grado de rapidísima obsolescencia de que adolece la maquinaria necesitada en cada caso (hardware).

En lo que se refiere a sus colecciones de materiales, cada vez es más difícil para una biblioteca nacional llegar a poseer todo el registro de las publicaciones del propio país, esencialmente debido al crecimiento en el número de las que continuamente no cesan de aparecer y también $-\mathrm{y}$ en no menor medida - debido a la imparable diversidad de los soportes y formatos.

Aunque podría pensarse que la venta de registros a otras bibliotecas ayudaría al sostenimiento de las bibliografías nacionales, la realidad ha demostrado que esto no es así. No obstante, conviene no olvidar que si el cumplimiento de las funciones tradicionales de una biblioteca nacional crea problemas, también genera oportunidades. El cobro por algunos servicios, tales como los de fotocopia, préstamo o información, puede ayudar a recabar fondos. Así mismo, también se puede conseguir financiación mediante las bases de datos bibliográficas o atendiendo a grupos especiales de usuarios que necesitan información derivada y procesada a partir de las colecciones. Algunas bibliotecas nacionales se han negado a cobrar por los servicios argumentando que establecerían un peligroso precedente; otras, como la British Library, razonan que de no ser así no se podrían mantener los servicios que prestan. Aún cuando muchas bibliotecas nacionales cobran por sus servicios, un problema añadido viene dado por el hecho de que en la mayoría de los casos los respectivos Gobiernos no les permiten manejar libremente las cantidades percibidas.

En cualquier caso, si las bibliotecas nacionales no reciben de sus Gobiernos la financiación adecuada, inevitablemente va a bajar su rendimiento, por lo que no les queda otro remedio que replantearse totalmente su modus operandi. El dilema ahora, como bien señala Line, es la elección entre mantenerse en la concha encogidos, o salir fuera. En el primer caso, y dada la creciente escasez de medios,

Scire. $9: 1$ (en.-jun. 2003) 57-74. 
la colección irá disminuyendo, de modo que a duras penas va a ser capaz de cumplir los objetivos tradicionales. En el segundo caso, si se produce la reacción adecuada de la biblioteca nacional, ésta será capaz de generar servicios dinámicos, basados en la colección, obteniendo como contrapartida dinero para el pago de estos servicios, así como para el desarrollo de la colección.

Esta cuestión de la autofinanciación por parte de las bibliotecas nacionales plantea, a su vez, el problema de si una institución que mayormente está financiada con fondos públicos puede o no competir con la empresa privada. En este sentido, Line plantea que una solución a estos problemas de carestía financiera podría ser ofrecer a las empresas privadas la explotación de los recursos de las colecciones, lo que en consecuencia podría generar productos vendibles de información comercial (Line, 1995). Algunas bibliotecas nacionales, sigue diciendo Line, ya están actuando en esta dirección, no sólo por las presiones de los Gobiernos sino también porque estas mismas bibliotecas nacionales son cada vez más conscientes de que necesitan protegerse y buscar alternativas a su erosión actual. "Hay riesgos, pero es mejor iniciar el viaje esperanzadamente que quedarse en casa".

Otra de las vías para la obtención de financiación a la que las bibliotecas nacionales están recurriendo cada vez con mayor dedicación es la que se refiere a la búsqueda de patrocinadores, sobre todo en cuanto a la adquisición de materiales para el aumento de sus colecciones. Esta vía viene a veces complementada con el alquiler de los espacios adecuados dentro de las instalaciones de la biblioteca al objeto de que se celebren en ellos eventos de importancia. Otro tanto sucede con la venta de reproducciones y objetos artísticos relacionados con los materiales más valiosos de las colecciones de estos centros (Smethurst, 2001, p. 132).

Al tratar la cuestión de la financiación de las bibliotecas nacionales resulta de enorme interés tomar en consideración las Pautas del Consejo de Europa sobre legislación y política bibliotecaria en Europa", por entender que suponen un adecuado planteamiento y unas buenas directrices. Dichas pautas fueron aprobadas en la $68^{\text {a }}$ Sesión del Consejo de Cooperación Cultural, celebrada los días 19 al 21 de enero del año 2000 (Guidelines..., 1998)

Conviene aclarar, en primer lugar, que estas Pautas están dirigidas a cualquier biblioteca, sea del tipo que sea, incluida en la política bibliotecaria del Consejo de Cooperación Cultural de Europa, por lo que resulta evidente que sus directrices, aunque no de manera exclusiva, también son aplicables a las bibliotecas nacionales europeas y, por ende, pueden servir como guía para cualquier biblioteca nacional de cualquier otro país del mundo (3).

Las Pautas comienzan con una Introducción, que recoge los principios inspiradores del documento, a la que siguen cuatro apartados sobre la libertad de expresión y libre acceso a la información, las bibliotecas en el marco de las políticas

Scire. $9: 1$ (en.-jun. 2003) 57-74. 
nacionales del libro y de la información, las bibliotecas y las industrias culturales, y la protección del patrimonio bibliotecario. De todo el conjunto de este documento, la parte que aquí nos interesa especialmente (a los efectos del presente trabajo) es la segunda, que trata de Las bibliotecas en el marco de las políticas nacionales del libro y de la información y, dentro de ella, el epígrafe 7, que tiene como título Financiación de las bibliotecas. Administración central y local. En él se establece claramente que la relación entre el nivel de servicio esperado y la dotación de los recursos adecuados para su prestación debe ser inequívoca, con independencia del tipo de biblioteca y el nivel del órgano administrativo que se ocupe de su gestión. En general, la financiación de las bibliotecas debe constituir una responsabilidad de la administración pública. Los servicios bibliotecarios que se financien con cargo a otras fuentes no deben influir en la integridad profesional de los bibliotecarios ni en la selección de los materiales de las bibliotecas, ni deben socavar los principios fundamentales de libre acceso ni la oferta gratuita de ciertos servicios básicos. Las autoridades responsables de las bibliotecas y las bibliotecas deben desarrollar conjuntamente estructuras organizativas y mecanismos de supervisión y control que garanticen la máxima rentabilidad posible de los fondos públicos dedicados a los servicios bibliotecarios, diseñando para ello instrumentos que permitan efectuar evaluaciones de rendimiento adecuadas y controlar la calidad de los diversos tipos de bibliotecas. Finalmente, los presupuestos de las bibliotecas deben reflejar la repercusión de las nuevas tecnologías y han de dotarse de recursos para garantizar una formación adecuada del personal y los usuarios para que puedan hacer un uso apropiado de las nuevas herramientas y servicios.

Queda, entendemos, suficientemente claro el pronunciamiento del Consejo de Europa acerca de la financiación de las bibliotecas, que se conciben como servicios públicos esenciales en un sistema democrático, ya que son las herramientas claves para el acceso libre y gratuito a la información de todos los ciudadanos.

Estas pautas, así mismo, señalan la responsabilidad de la administración pública en la financiación de la biblioteca, así como la necesidad de una actuación conjunta entre las bibliotecas y las correspondientes administraciones al objeto de conseguir la máxima rentabilidad posible de los fondos públicos, incidiéndose así mismo en la importancia de las evaluaciones de rendimientos adecuadas y del control de la calidad de las bibliotecas. También llaman expresamente la atención respecto a la adecuada financiación de las nuevas tecnologías, con las partidas necesarias y suficientes que garanticen la formación adecuada del personal y de los usuarios en el uso de estas nuevas herramientas y servicios.

\section{Notas}

(1) Ineludiblemente unido al concepto de planificación aparece el de calidad (Pinto Molina y Sánchez Ambriz, 1997).

Scire. $9: 1$ (en.-jun. 2003) 57-74. 


\section{4}

(2) En este mismo sentido de la enormidad de gasto que supone cualquier intervención arquitectónica en el edificio de una biblioteca nacional -y ahora no nos estamos refiriendo a la construcción de un nuevo edificio - conviene señalar el hecho de que la Biblioteca Nacional de España tuvo en 1998 un incremento presupuestario del 46,6 \% respecto al año anterior, que fue totalmente absorbido por las obras de remodelación del edificio (Smethurst, 2001, p. 129).

(3) Como comentario al origen de estas pautas, véase Arot (1999, p. 98-99).

\section{Referencias}

Arot, Dominique (1999). Le local et le global dans la legislation sur les bibliothéques. // Bulletin des Bibliothéques de France. 44:4 (1999) 98-99.

Ferguson, Stephney (1992). Strategic planning for national libraries in developing countries: an optimist's view. // IFLA Journal. 18:4 (1992) 339-344.

Fuentes Romero, Juan José (2001). Planificación de centros bibliotecarios. // Revista General de Información y Documentación. 11:1 (2001) 80.

Guidelines on Library Legislation and Policy in Europe: Strasbourg, 23-25 November 1998 / Council of Europe; EBLIDA. URL:< http://www.coe.int/T/E/Cultural_Cooperation/Culture/Resources/Reference_texts/Guidelines/ecubook_R3.asp\#P135_ 27323 > Consultado: 02-02-2004.

Kast, F. E.; Rosenzweig, J. E. (1987). Administración en las organizaciones: Enfoque de sistemas y contingencias. México: Mc Graw-Hill, 1987.

Line, Maurice B. (1991). Strategic planning in national libraries. Paper presented at the 57th. IFLA General Conference, 18-24 August, 1991.

Line, Maurice B. (1995). National libraries in a time of change. // Line, Maurice B.; Line, Joyce (eds.). National Libraries: A selection of articles on national libraries, 19861994. London: ASLIB, 1995.

Line, Maurice B. (1995) Part II: Special aspects. // Line, Maurice B.; Line, Joyce (eds.). National Libraries: A selection of articles on national libraries, 1986-1994. London: ASLIB, 1995.

Mintzberg, Henry (1994). The rise and fall of strategic planning. New York: Prentice Hall, 1994.

National Library of Canada (1998). Core competencies. Ottawa: National Library of Canada, 1998. URL: http://www.ifla.org/VII/s1/news/dec/1998.html . Consultado: 28-01-2002)

Pinto Molina, María; Sánchez Ambriz, Gerardo (1997). Planificación estratégica y calidad en los sistemas de información científica. // Ciencias de la Información. 28:4 (1997) 241-249.

Revista General de Información y Documentación. 11:1 (2001) 80.

Smethurst, Michael (2001). European national libraries: a review of the year's activities 1999-2000. // Liber Quarterly. 11:2 (2001) 129.

Steiner, George (1969). Top management planning. New York: Macmillan, 1969. 